Supplement of Biogeosciences, 18, 2487-2510, 2021 https://doi.org/10.5194/bg-18-2487-2021-supplement (C) Author(s) 2021. CC BY 4.0 License.

(c) (1)

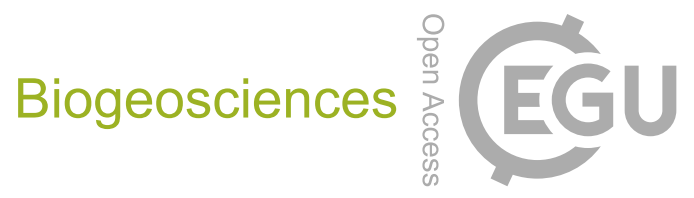

Supplement of

\title{
Isoprene and monoterpene emissions from alder, aspen and spruce short- rotation forest plantations in the United Kingdom
}

\section{Gemma Purser et al.}

Correspondence to: Gemma Purser (gemma.purser1 @ gmail.com)

The copyright of individual parts of the supplement might differ from the article licence. 
Figure S1 - Meteorological data measured during the chamber sampling campaign from May 2018 until July 2019 at the East Grange SRF trial site. (Occasional missing data due to power failure at the site.) Rainfall data was provided by SEPA for the Saline rainfall monitoring station $\left(56.115^{\circ} \mathrm{N}\right.$ $3.574^{\circ} \mathrm{W}$ ), approximately $4 \mathrm{~km}$ north-east of East Grange.
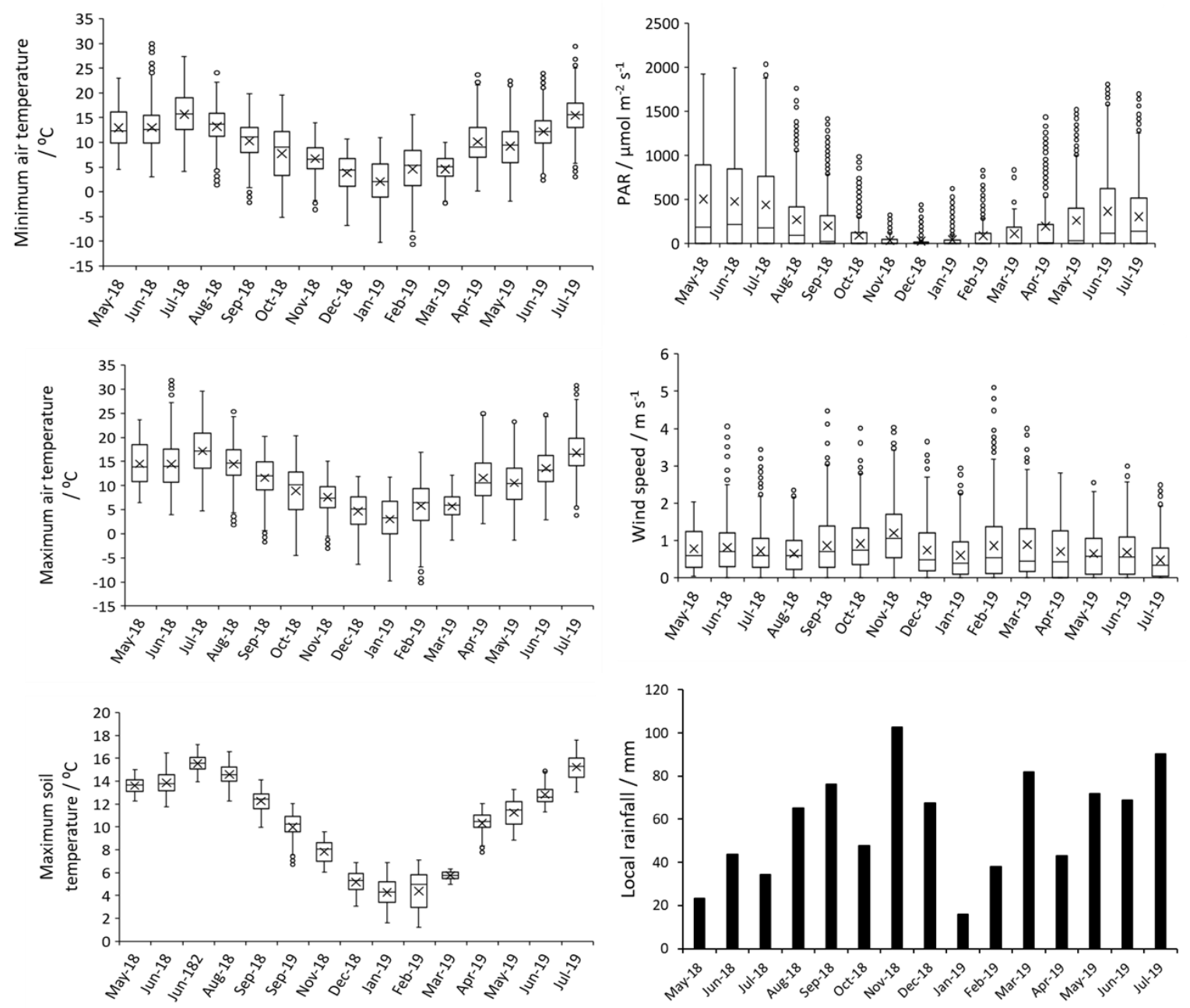
Figure S2 - Summary data for PAR and air temperature measured at the East Grange field site, Fife, Scotland. Missing data was gap filled with Easter Bush field data, Midlothian, Scotland and the summary data presented here reflects this. The data represented here was used for modelling annual BVOC emissions from Sitka spruce, hybrid aspen and Italian alder using the MEGAN 2.1 model.

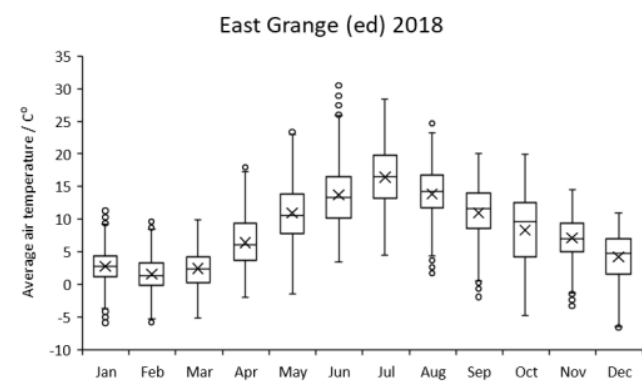

East Grange (gap filled) 2019

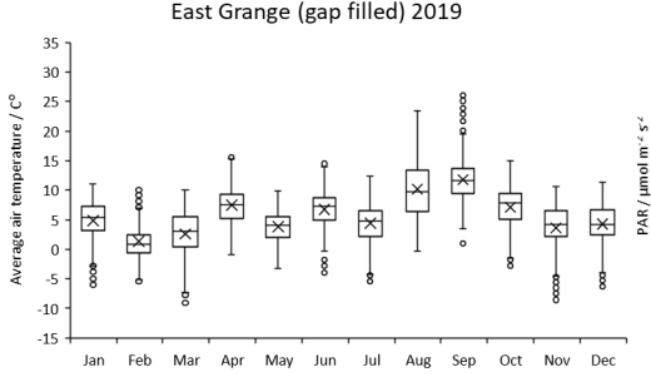

East Grange (gap filled) 2018

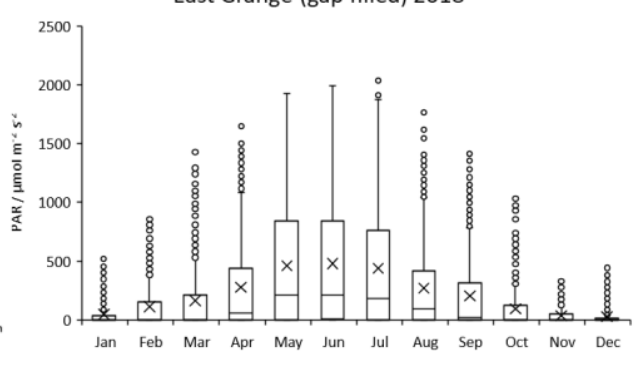

East Grange (gap filled) 2019

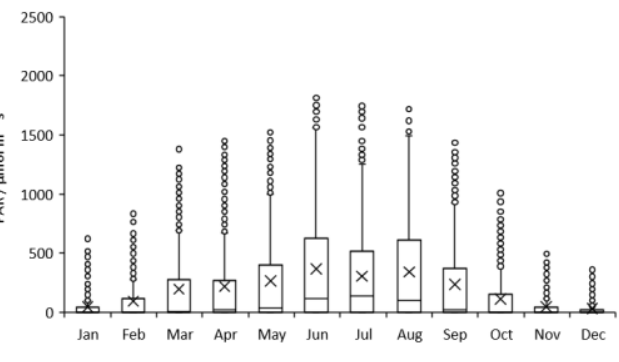


Figure S3 - Summary data for PAR and air temperature measured at the Alice Holt field site, south England. This data was used for modelling annual BVOC emissions from Sitka spruce, hybrid aspen and Italian alder using the MEGAN 2.1 model.
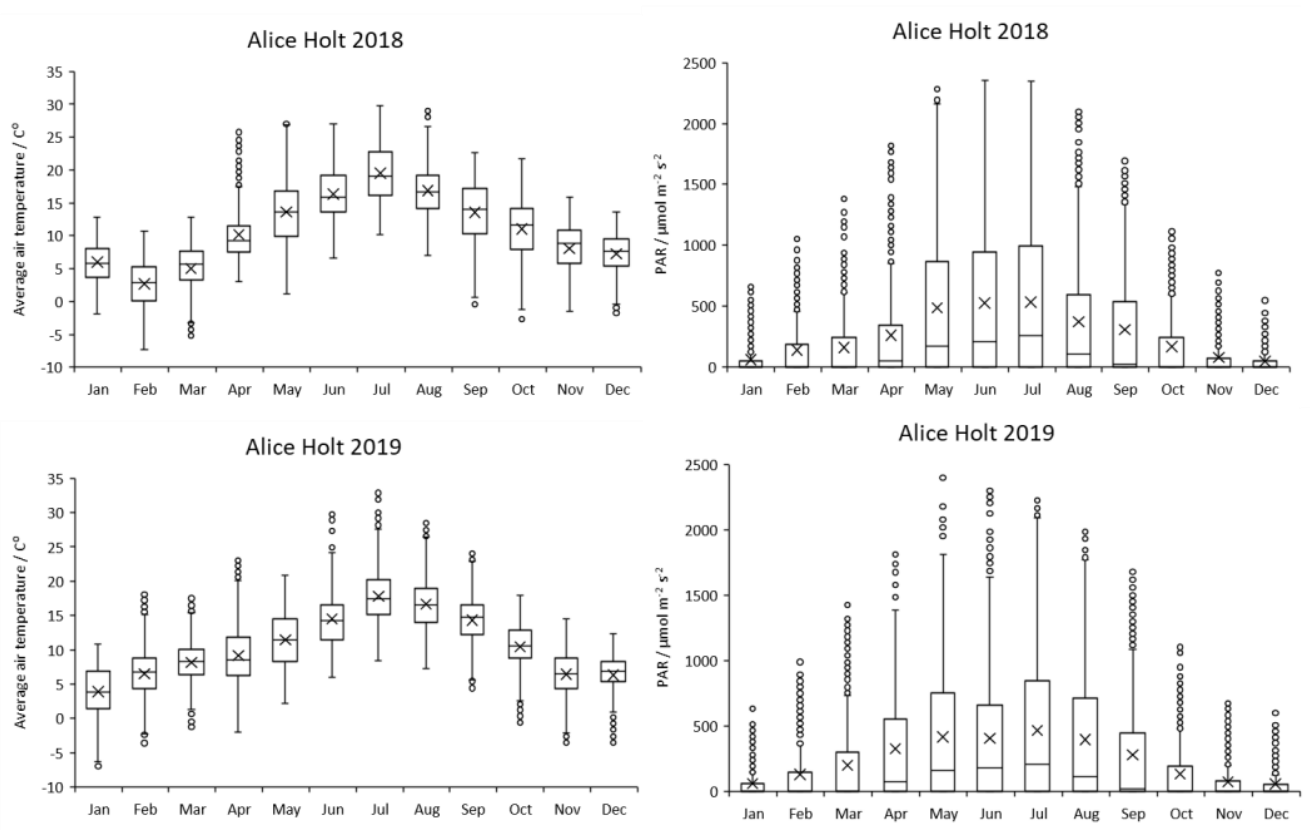
Table S1 - Median seasonal BVOC emissions $\left(\mu \mathrm{g} \mathrm{g} \mathrm{g}^{-1} \mathrm{~h}^{-1}\right)$ and corresponding interquartile range from branches of Sitka spruce, hybrid aspen and Italian alder in SRF plantations, East Grange, Fife, Scotland.

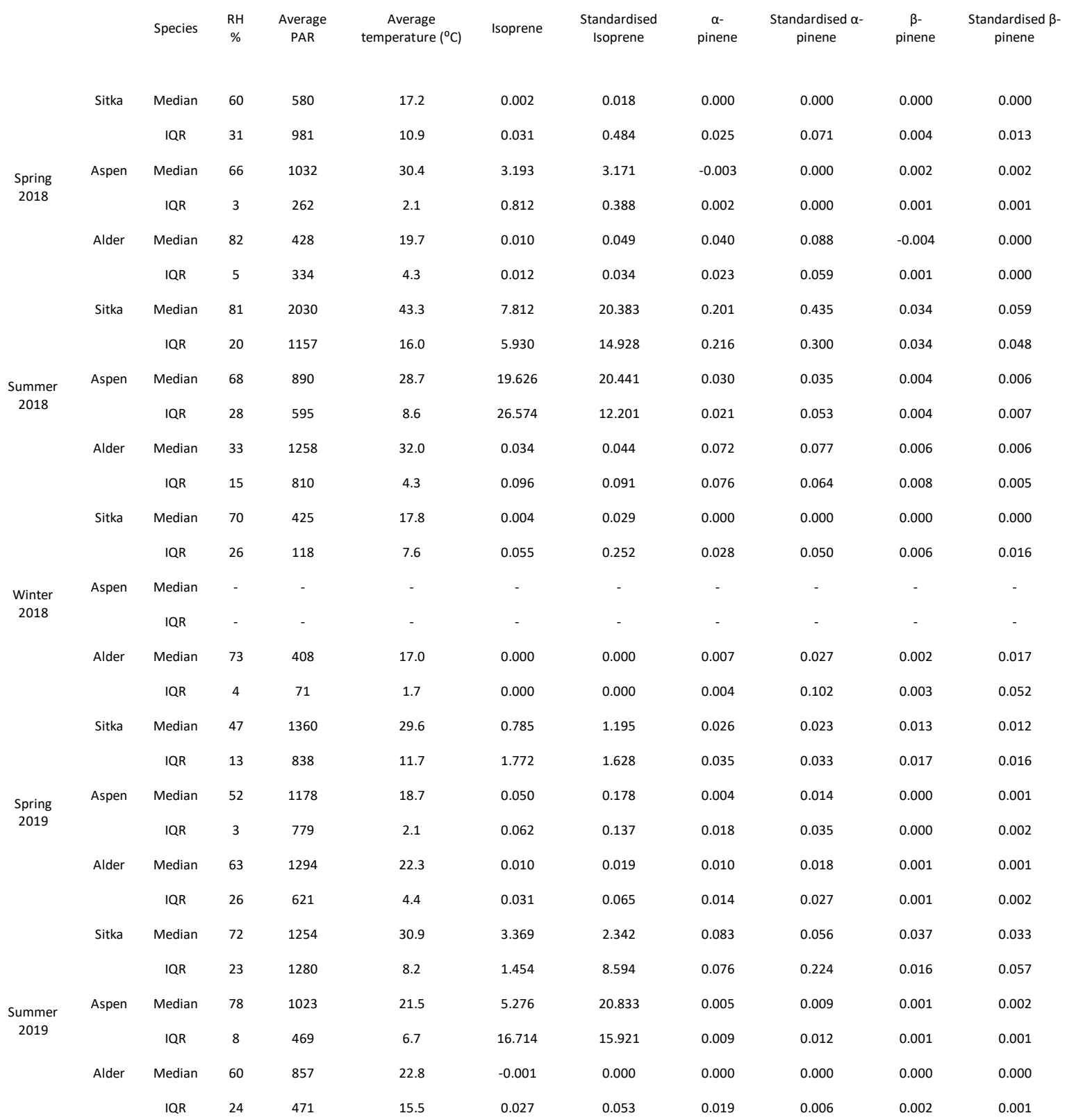




\section{Table S1 continued}

\begin{tabular}{|c|c|c|c|c|c|c|c|c|c|c|c|c|}
\hline & & Species & camphene & $\begin{array}{l}\text { Standardised } \\
\text { camphene }\end{array}$ & $\begin{array}{c}\beta- \\
\text { myrcene }\end{array}$ & $\begin{array}{c}\text { Standardised } \\
\beta \text {-myrcene }\end{array}$ & $\begin{array}{c}\alpha- \\
\text { phellandrene }\end{array}$ & $\begin{array}{c}\text { Standardised } \\
\alpha- \\
\text { phellandrene }\end{array}$ & $\begin{array}{c}\beta- \\
\text { phellandrene }\end{array}$ & $\begin{array}{c}\text { Standardised } \\
\beta- \\
\text { phellandrene }\end{array}$ & $\begin{array}{c}d- \\
\text { limonene }\end{array}$ & $\begin{array}{l}\text { Standardised } \\
\text { d-limonene }\end{array}$ \\
\hline \multirow{6}{*}{$\begin{array}{c}\text { Spring } \\
2018\end{array}$} & Sitka & Median & 0.000 & 0.000 & 0.000 & 0.000 & 0.000 & 0 & 0.000 & 0 & 0.000 & 0 \\
\hline & & IQR & 0.022 & 0.064 & 0.044 & 0.130 & 0.000 & 0.000 & 0.000 & 0.000 & 0.033 & 0.098 \\
\hline & Aspen & Median & 0.002 & 0.002 & 0.019 & 0.017 & 0.000 & 0.000 & 0.000 & 0.000 & 0.053 & 0.052 \\
\hline & & IQR & 0.001 & 0.002 & 0.020 & 0.016 & 0.000 & 0.000 & 0.000 & 0.000 & 0.017 & 0.029 \\
\hline & Alder & Median & 0.001 & 0.003 & 0.022 & 0.052 & 0.000 & 0.001 & 0.000 & 0.000 & 0.132 & 0.334 \\
\hline & & IQR & 0.006 & 0.009 & 0.007 & 0.012 & 0.001 & 0.002 & 0.000 & 0.000 & 0.084 & 0.388 \\
\hline \multirow{6}{*}{$\begin{array}{c}\text { Summer } \\
2018\end{array}$} & Sitka & Median & 0.205 & 0.450 & 8.000 & 13.314 & 0.033 & 0.073 & 0.028 & 0.061 & 0.530 & 1.089 \\
\hline & & IQR & 0.190 & 0.234 & 0.972 & 1.502 & 0.016 & 0.025 & 0.022 & 0.035 & 0.555 & 0.843 \\
\hline & Aspen & Median & 0.003 & 0.005 & 0.007 & 0.015 & 0.000 & 0.000 & 0.004 & 0.009 & 0.045 & 0.045 \\
\hline & & IQR & 0.008 & 0.012 & 0.010 & 0.016 & 0.000 & 0.000 & 0.009 & 0.010 & 0.064 & 0.035 \\
\hline & Alder & Median & 0.015 & 0.052 & 0.051 & 0.233 & 0.000 & 0.000 & 0.000 & 0.000 & 0.037 & 0.051 \\
\hline & & IQR & 0.071 & 0.061 & 0.278 & 0.240 & 0.000 & 0.000 & 0.000 & 0.000 & 0.146 & 0.105 \\
\hline \multirow{6}{*}{$\begin{array}{c}\text { Winter } \\
2018\end{array}$} & Sitka & Median & 0.000 & 0.000 & 0.003 & 0.011 & 0.000 & 0.000 & 0.000 & 0.000 & 0.005 & 0.017 \\
\hline & & IQR & 0.011 & 0.017 & 0.104 & 0.300 & 0.001 & 0.001 & 0.000 & 0.001 & 0.060 & 0.178 \\
\hline & Aspen & Median & - & - & - & - & - & - & - & - & - & - \\
\hline & & IQR & - & - & - & - & - & - & - & - & - & - \\
\hline & Alder & Median & 0.000 & 0.000 & 0.001 & 0.010 & 0.000 & 0.002 & 0.000 & 0.000 & 0.025 & 0.196 \\
\hline & & IQR & 0.000 & 0.000 & 0.002 & 0.003 & 0.000 & 0.001 & 0.000 & 0.000 & 0.018 & 0.369 \\
\hline \multirow{6}{*}{$\begin{array}{c}\text { Spring } \\
2019\end{array}$} & Sitka & Median & 0.010 & 0.009 & 0.916 & 0.834 & 0.002 & 0.003 & 0.006 & 0.006 & 0.430 & 0.392 \\
\hline & & IQR & 0.012 & 0.010 & 1.188 & 1.340 & 0.006 & 0.006 & 0.009 & 0.008 & 0.524 & 0.476 \\
\hline & Aspen & Median & 0.000 & 0.000 & 0.000 & 0.000 & 0.000 & 0.000 & 0.000 & 0.000 & 0.000 & 0.000 \\
\hline & & IQR & 0.000 & 0.000 & 0.000 & 0.001 & 0.000 & 0.000 & 0.000 & 0.000 & 0.004 & 0.009 \\
\hline & Alder & Median & 0.000 & 0.000 & 0.001 & 0.001 & 0.000 & 0.000 & 0.000 & 0.001 & 0.013 & 0.018 \\
\hline & & IQR & 0.000 & 0.000 & 0.001 & 0.001 & 0.000 & 0.000 & 0.000 & 0.001 & 0.016 & 0.033 \\
\hline \multirow{6}{*}{$\begin{array}{l}\text { Summer } \\
2019\end{array}$} & Sitka & Median & 0.005 & 0.005 & 1.046 & 0.786 & 0.012 & 0.012 & 0.020 & 0.013 & 0.719 & 0.742 \\
\hline & & IQR & 0.052 & 0.111 & 0.756 & 0.383 & 0.009 & 0.008 & 0.016 & 0.009 & 0.233 & 0.874 \\
\hline & Aspen & Median & 0.000 & 0.000 & 0.000 & 0.001 & 0.000 & 0.000 & 0.001 & 0.001 & 0.009 & 0.023 \\
\hline & & IQR & 0.001 & 0.001 & 0.001 & 0.001 & 0.000 & 0.000 & 0.011 & 0.013 & 0.015 & 0.016 \\
\hline & Alder & Median & 0.000 & 0.000 & 0.000 & 0.000 & 0.000 & 0.000 & 0.000 & 0.000 & 0.004 & 0.005 \\
\hline & & IQR & 0.001 & 0.000 & 0.000 & 0.001 & 0.000 & 0.000 & 0.006 & 0.000 & 0.004 & 0.012 \\
\hline
\end{tabular}




\section{Table S1 continued}

\begin{tabular}{|c|c|c|c|c|c|c|c|c|c|c|c|c|}
\hline & & Species & eucalyptol & $\begin{array}{l}\text { Standardised } \\
\text { eucalyptol }\end{array}$ & Carene & $\begin{array}{l}\text { Standardised } \\
\text { carene }\end{array}$ & linalool & $\begin{array}{l}\text { Standardised } \\
\text { linalool }\end{array}$ & $\begin{array}{c}Y^{-} \\
\text {terpinene }\end{array}$ & $\begin{array}{c}\text { Standardised } \\
\gamma \text {-terpinene }\end{array}$ & Total MT & $\begin{array}{c}\text { Standardised } \\
\text { Total MT }\end{array}$ \\
\hline \multirow{6}{*}{$\begin{array}{c}\text { Spring } \\
2018\end{array}$} & Sitka & Median & 0.000 & 0 & 0.000 & 0 & 0.000 & 0 & 0.000 & 0 & 0.000 & 0.000 \\
\hline & & IQR & 0.001 & 0.004 & 0.000 & 0.000 & 0.000 & 0.000 & 0.000 & 0.000 & 0.127 & 0.436 \\
\hline & Aspen & Median & 0.008 & 0.008 & -0.003 & 0.000 & 0.000 & 0.000 & 0.000 & 0.000 & 0.065 & 0.074 \\
\hline & & IQR & 0.004 & 0.003 & 0.001 & 0.000 & 0.000 & 0.000 & 0.000 & 0.000 & 0.014 & 0.016 \\
\hline & Alder & Median & 0.005 & 0.010 & 0.036 & 0.089 & 0.000 & 0.000 & 0.000 & 0.000 & 0.232 & 0.584 \\
\hline & & IQR & 0.001 & 0.005 & 0.006 & 0.044 & 0.000 & 0.000 & 0.000 & 0.000 & 0.085 & 0.531 \\
\hline \multirow{6}{*}{$\begin{array}{l}\text { Summer } \\
2018\end{array}$} & Sitka & Median & 0.072 & 0.158 & 0.011 & 0.019 & 0.000 & 0.000 & 0.000 & 0.000 & 9.025 & 15.021 \\
\hline & & IQR & 0.193 & 0.097 & 0.016 & 0.014 & 0.000 & 0.000 & 0.000 & 0.000 & 2.270 & 3.136 \\
\hline & Aspen & Median & 0.008 & 0.012 & 0.013 & 0.020 & 0.000 & 0.000 & 0.000 & 0.000 & 0.107 & 0.157 \\
\hline & & IQR & 0.013 & 0.025 & 0.018 & 0.011 & 0.000 & 0.000 & 0.000 & 0.000 & 0.131 & 0.101 \\
\hline & Alder & Median & 0.016 & 0.021 & 0.025 & 0.031 & 0.000 & 0.000 & 0.000 & 0.000 & 0.387 & 0.435 \\
\hline & & IQR & 0.015 & 0.009 & 0.059 & 0.058 & 0.000 & 0.000 & 0.000 & 0.000 & 0.563 & 0.483 \\
\hline \multirow{6}{*}{$\begin{array}{l}\text { Winter } \\
2018\end{array}$} & Sitka & Median & 0.000 & 0.000 & 0.000 & 0.001 & 0.000 & 0.000 & 0.000 & 0.000 & 0.008 & 0.025 \\
\hline & & IQR & 0.022 & 0.038 & 0.003 & 0.009 & 0.001 & 0.001 & 0.000 & 0.000 & 0.258 & 0.741 \\
\hline & Aspen & Median & - & - & - & - & - & - & - & - & - & - \\
\hline & & IQR & - & - & - & - & - & - & - & - & - & - \\
\hline & Alder & Median & 0.001 & 0.007 & 0.006 & 0.037 & 0.000 & 0.002 & 0.000 & 0.001 & 0.037 & 0.342 \\
\hline & & IQR & 0.001 & 0.012 & 0.004 & 0.098 & 0.000 & 0.003 & 0.000 & 0.002 & 0.021 & 0.658 \\
\hline \multirow{6}{*}{$\begin{array}{l}\text { Spring } \\
2019\end{array}$} & Sitka & Median & 0.000 & 0.000 & 0.006 & 0.007 & 0.000 & 0.000 & 0.000 & 0.000 & 1.412 & 1.286 \\
\hline & & IQR & 0.022 & 0.044 & 0.011 & 0.011 & 0.000 & 0.000 & 0.000 & 0.000 & 2.391 & 2.602 \\
\hline & Aspen & Median & 0.000 & 0.000 & 0.001 & 0.002 & 0.000 & 0.000 & 0.000 & 0.000 & 0.005 & 0.020 \\
\hline & & IQR & 0.000 & 0.000 & 0.001 & 0.002 & 0.007 & 0.018 & 0.000 & 0.000 & 0.071 & 0.137 \\
\hline & Alder & Median & 0.000 & 0.000 & 0.005 & 0.010 & 0.001 & 0.001 & 0.000 & 0.000 & 0.035 & 0.051 \\
\hline & & IQR & 0.000 & 0.000 & 0.008 & 0.009 & 0.002 & 0.004 & 0.000 & 0.000 & 0.038 & 0.085 \\
\hline \multirow{6}{*}{$\begin{array}{l}\text { Summer } \\
2019\end{array}$} & Sitka & Median & 0.073 & 0.072 & 0.008 & 0.011 & 0.005 & 0.005 & 0.003 & 0.003 & 2.302 & 2.057 \\
\hline & & IQR & 0.136 & 0.048 & 0.023 & 0.009 & 0.010 & 0.004 & 0.005 & 0.002 & 1.160 & 1.460 \\
\hline & Aspen & Median & 0.000 & 0.000 & 0.003 & 0.007 & 0.004 & 0.011 & 0.000 & 0.000 & 0.023 & 0.049 \\
\hline & & IQR & 0.006 & 0.007 & 0.006 & 0.008 & 0.037 & 0.042 & 0.000 & 0.000 & 0.081 & 0.094 \\
\hline & Alder & Median & 0.000 & 0.000 & 0.000 & 0.000 & 0.000 & 0.000 & 0.000 & 0.000 & 0.004 & 0.014 \\
\hline & & IQR & 0.000 & 0.000 & 0.009 & 0.004 & 0.000 & 0.000 & 0.000 & 0.000 & 0.024 & 0.017 \\
\hline
\end{tabular}

